\title{
Tagung: Internationale Freiwilligendienste im Spiegel des demografischen Wandels
}

\author{
Jennifer Neumann \\ Dipl. Reg.-Wiss. Lateinamerika | für den Arbeitskreis Lernen und \\ Helfen in Übersee (AKLHÜ) e. V. | jenny.neumann@posteo.de
}

\section{Einführung und Überblick}

Der demografische Wandel mit seinen Implikationen für die zukünftige Altersstruktur der Gesellschaft stellt die Entwicklung internationaler Freiwilligendienste vor neue Herausforderungen. Gemäß den Berechnungen des statistischen Bundesamts führt das stabil niedrige Geburtenniveau bei gleichzeitig ansteigender Lebenserwartung zu gravierenden Verschiebungen in der Bevölkerungsstruktur. Die Folge: Im Jahr 2050 wird jeder Dritte bereits über 65 Jahre alt sein, der Anteil der unter 20-Jährigen liegt bei nur 15 Prozent. Verschärft wird diese Entwicklung durch den aktuellen Altersaufbau mit einem hohen Anteil von Menschen im mittleren Alter. Alle Faktoren zusammen genommen führen dazu, dass sich das Generationenverhältnis weiterhin zu Lasten der Jüngeren verschieben wird - ein Trend, der auch mit prognostizierten Zuwanderungsströmen von 100.000 bis 200.000 Personen pro Jahr nicht aufgefangen werden kann (Statistisches Bundesamt 2009: 5f.).

Neben den genannten Folgen für die deutsche Gesellschaft, wirkt sich die veränderte Bevölkerungsstruktur auch auf die „klassischen“ Zielgruppen internationaler Freiwilligendienste aus. Kurz- bis mittelfristig wird ein Rückgang der Teilnehmerzahlen junger Menschen zwischen 18 und 28 Jahren erwartet, sodass Berufstätige, über 30-Jährige und ältere Menschen sowie ausländische TeilnehmerInnen als neue Zielgruppen für internationale Dienste in Frage kommen und eine inhaltliche Anpassung der bestehenden Formate stattfinden muss.

Vor dem Hintergrund dieser Entwicklung fand am 5. und 6. Mai 2014 im Katholisch-Sozialen Institut in Bad Honnef die Tagung „Alle reden vom demografischen Wandel - Brauchen internationale Freiwilligendienste neue Perspektiven?" statt. 80 MitarbeiterInnen von Trägerorganisationen sowie VertreterInnen der verschiedenen Qualitätsstellen, Dachverbände, der Bundesministerien für Familie, Senioren, Frauen und Jugend (BMFSFJ) und für wirtschaftliche Zusammenarbeit und Entwicklung (BMZ) kamen zusammen, um über aktuelle Auswirkungen des demografischen Wandels auf internationale Freiwilligendienste zu diskutieren und gemeinsam nach neuen Impulsen für die zukünftige Arbeit zu suchen. ${ }^{1}$

\footnotetext{
1 Dieser Artikel basiert im Wesentlichen auf den Ergebnissen und Dokumentation der Perspektivkonferenz "Alle reden vom demografischen Wandel - Brauchen internationale Freiwilligendienste neue Perspektiven?", 5. und 6. Mai, Bad Honnef), ergänzt um weiteres Literatur- und Quellenmaterial. Alle genannten Ergebnisse oder Empfehlungen beziehen sich auf getätigte Aussagen während der Konferenz, aufbereitet und redaktionell bearbeitet durch die Autorin und den AKLHÜ.
} 
Im Fokus der von den Zentralen Stellen im Internationalen Jugendfreiwilligendienst (IJFD) ${ }^{2}$ ausgerichteten Perspektivkonferenz standen folgende Themen:

1. Die Erweiterung der Zielgruppen in internationalen Freiwilligendiensten durch die im Zuge der G8-Reform verkürzten Schulzeiten,

2. die stärkere Öffnung bestehender Angebote für über 30-Jährige und ältere Menschen,

3. die Ausweitung betrieblicher Freiwilligenprogramme im Rahmen von Corporate Volunteering sowie mögliche Anreize und Anknüpfungspunkte für Kooperationen in diesem Bereich und schließlich

4. der quantitative Ausbau und die konzeptionelle Weiterentwicklung der Incoming-Programme, damit verbundene Anforderungen an Einsatzstellen, Träger- und Entsendeorganisationen sowie politische und finanzielle Rahmenbedingungen.

Die folgende Grafik fasst die einzelnen „Säulen“ der Tagung und ihre Handlungsempfehlungen zusammen:

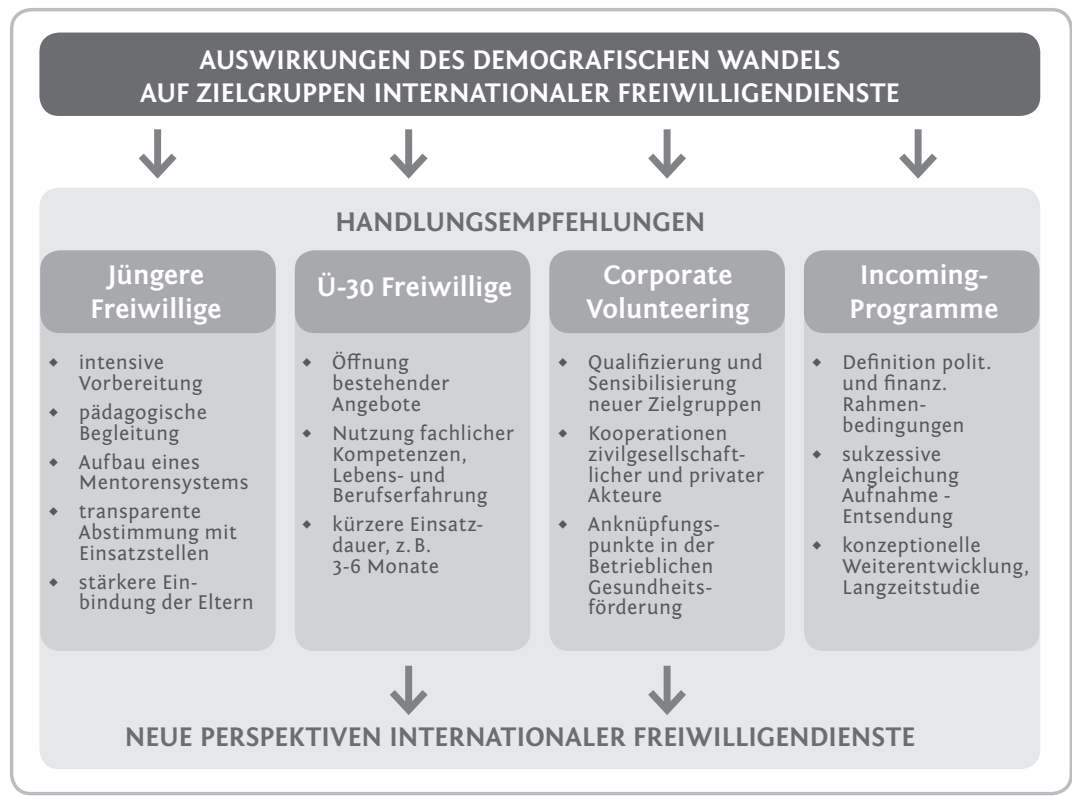

Quelle: Eigene Darstellung

2 Die „Zentralen Stellen“ im Internationalen Jugendfreiwilligendienst haben den Auftrag, die Qualitätssicherung und -entwicklung sowie die Vernetzung der angeschlossenen Träger zu unterstützen. Sie bündeln und sichern zudem die Kommunikation zwischen dem BMFSFJ und den Trägern. 


\section{Erweiterung der Zielgruppen in internationalen Freiwilligendiensten}

In einem grundlegenden Vortrag stellte Christoph Krauß (wissenschaftlicher Mitarbeiter, Katholische Sozialwissenschaftliche Zentralstelle) zu Beginn die Folgen des demografischen Wandels auf die Engagementbereitschaft der Bevölkerung dar. Resultate wie eine Überalterung der Gesellschaft können langfristig das herkömmliche Engagementpotential mindern. Zusammen mit dem verstärkten Druck zum schnellen Weg auf den Arbeitsmarkt wird so das „klassische“ Potential für Freiwilligendienste, besonders im Ausland, eingeschränkt. Somit bedarf es einer Erweiterung der Zielgruppen und damit neuer Wege, Jüngere, Ältere, Berufstätige und auch bislang nicht Engagierte zu gewinnen.

\subsection{Internationale Freiwilligendienste für junge Menschen}

Ein Auslandsjahr oder auch ein kürzerer Aufenthalt von einigen Monaten ist der Wunsch vieler Jugendlicher, die ihre Schulausbildung abgeschlossen haben und mit dem Studium oder der beruflichen Ausbildung noch nicht beginnen wollen. Durch die verkürzte Schulzeit werden die InteressentInnen für internationale Dienste immer jünger. Die Entsendung von jungen Menschen, insbesondere unter 18-Jähriger (U-18), stellt nicht nur die Trägerorganisationen, sondern auch die lokalen Partnerorganisationen im Ausland vor neue Herausforderungen. Die jungen Freiwilligen gehen mit anderen Erwartungen und Fähigkeiten in den internationalen Dienst.

Die Prüfung der emotionalen Reife, Selbstständigkeit und Belastbarkeit sowie der persönlichen Motive sind in der Vorauswahl von großer Bedeutung ebenso wie die intensive Vorbereitung der angehenden Freiwilligen auf Basis eines qualifizierten und auf die individuellen Bedürfnisse der jüngeren TeilnehmerInnen zugeschnittenen Seminarkonzepts. Dies setzt voraus, dass die im Inland eingebundenen SeminarleiterInnen und MentorInnen sowie die AnsprechpartnerInnen der Partnerorganisationen, im Rahmen von Fort- und Weiterbildungen, pädagogisch auf die neue Zielgruppe vorbereitet werden müssen.

Neben der Klärung der rechtlichen Rahmenbedingungen für die Entsendung von U-18-BewerberInnen ist es erforderlich, die Eltern als Erziehungsberechtigte verstärkt in den Vorbereitungsprozess einzubinden. Der erhöhte Bedarf an persönlicher Zuwendung und Einführung, die Aufrechterhaltung fortwährender Kontakte mit den pädagogischen BetreuerInnen in Deutschland und die damit einhergehende notwendige Steigerung der personalen und finanziellen Ressourcen können als Ergebnisse des ersten Fachkonferenztages festgehalten werden. Darüber hinaus spielt auch der Umgang mit neuen Lebensrealitäten, speziell in der Nutzung von Internet und sozialen Netzwerken, als Querschnittsthema in diesem Kontext 
eine große Rolle. Wie Rüdiger Sweere (Referent, OUTtakes Bonn) darlegte, ist die tägliche Verfügbarkeit des Internets auch während des Freiwilligendienstes für viele junge Menschen essentiell, sodass sich Entsende- wie Aufnahmeorganisationen in diesem Feld auf neue Entwicklungen einstellen müssen.

Die Einbeziehung der Einsatzstellen als gleichberechtigte Partner in den Entscheidungsprozess um eine Entsendung jüngerer Freiwilliger ist unabdingbar. Eine Sammlung von ausgewählten Statements der Partnerorganisationen hat ergeben, dass die tendenzielle Verjüngung der Freiwilligen in internationalen Diensten zum Teil kritisch gesehen werde. Es wird befürchtet, dass der erhöhte Bedarf an Fürsorge zulasten der Aufgaben- und Rollenorientierung gehe und mangelndes Reflexionsvermögen die interkulturelle Kommunikation zusätzlich belasten könne. Aus diesen Überlegungen ergibt sich, dass für junge Zielgruppen eine intensive Vorbereitungsphase besonders wichtig ist, um einen kritischen Umgang mit bestehenden Vorurteilen und Stereotypen zu erlernen, die Reflexion der eigenen Rolle anzustoßen und der Reproduktion rassistischer und neokolonialer Bilder vorzubeugen.

Darüber hinaus wurde auf der Tagung der Aufbau eines Mentoringsystems in Erwägung gezogen, bei dem jüngere Freiwillige gemeinsam mit einem älteren Freiwilligen entsendet werden. Eine weitere Variante könnte die Einbindung zurückgekehrter Freiwillige vorsehen, die den Jüngeren bereits in der Vorbereitung im Rahmen eines Tandems zur Seite stehen und diese auch während des Einsatzes über das Internet als MentorInnen begleiten.

Eine potentielle Öffnung bestehender Angebote für jüngere Menschen erfordert folglich klare politische und rechtliche Rahmenbedingungen, intensive Vorbereitung und pädagogische Begleitung sowie transparente Absprachen mit den Partnern - wobei unabhängig davon, ob es sich um minder- oder volljährige TeilnehmerInnen handelt, die persönliche Reife in allen Phasen des Einsatzes stets im Vordergrund stehen sollte.

\subsection{Internationale Freiwilligendienste für Ü-30-Jährige und ältere Menschen}

In der Diskussion um eine Erweiterung der Zielgruppen zählte auch der Austausch zu Angeboten für Freiwillige über 30 Jahren (Ü-30) zu den Kernthemen der Perspektivkonferenz. Unter dem Titel „Zu alt, zu geprägt und zu unflexibel“ oder „Reif, erfahren und weltgewandt?“ sprachen Elisabeth Freise (Freiwilligenkoordinatorin, EIRENE), Bettina Hartmann (Leiterin Abteilung Experten, Senior Experten Service) und Wolfram Hahnfeld (ehemaliger ,älterer“ Freiwilliger) über passende Formate eines internationalen Dienstes für die ältere Zielgruppe.

Mit Ausnahme einzelner Programme sind die Möglichkeiten zur Teilnahme an internationalen Einsätzen für die mittlere und ältere Generation bisher 
eingeschränkt und für Freiwillige über 30 Jahren ist es - jenseits von Fachkräfteeinsätzen innerhalb der Entwicklungszusammenarbeit - aufgrund von Altersbeschränkungen zunehmend schwer, sich im Ausland zu engagieren (zze 2007: 36). Angesichts der demografischen Entwicklung und der steigenden Lebenserwartung älterer Menschen ist es jedoch wichtig, die Angebote auch für diese Zielgruppe zu öffnen. Ausgeprägte fachliche Kompetenzen sowie Lebens- und Berufserfahrung ermöglichen es den Älteren, andere Impulse zu setzen als junge Menschen und dabei auf ein umfangreiches Erfahrungswissen zurückzugreifen. Durch eine stärkere Einbindung dieser Zielgruppe gelingt es, die Potentiale der älteren Generationen umfassender zu nutzen und ihr Wissen und Know-How in die Gesellschaft einzubringen. Die Grundmotivation älterer Freiwilliger weist dabei Parallelen zu den Beweggründen der Jüngeren auf: Sie entscheiden sich für einen Freiwilligendienst, um „etwas Gutes zu tun“, aber auch um ihrem Wunsch nach Neu- oder Umorientierung nachzugehen, eine Auszeit von Familie oder Beruf zu nehmen oder die freie Zeit im Ruhestand sinnvoll zu nutzen.

Doch die Frage nach einem Ausbau und möglichen Formaten internationaler Freiwilligendienste für über 30-Jährige hat auch kritische Einwände auf der Tagung hervorgerufen. In der Diskussion standen u. a. die spezifischen Erwartungen dieser Zielgruppe an einen internationalen Dienst, z. B. bei der Überbrückung von Arbeitslosigkeit oder als Perspektive für den beruflichen Wiedereinstieg, ebenso wie die inhaltliche Ausgestaltung der Programme in Bezug auf Qualifizierung, Vorbereitung und pädagogische Begleitung der Ü-30-Teilnehmenden. Insbesondere in diesem letzten Punkt stellte sich auch die Frage, welche Modelle für ältere Freiwillige geeignet seien und inwiefern ein Freiwilligendienst für diese Zielgruppe Berufserfahrungen und fachliche Kenntnisse einbeziehen soll, die bei Programmen für die jüngere Generation nicht von Relevanz sind.

Als Fazit der Podiumsdiskussion kann festgehalten werden: Die Nachfrage der Älteren ist hoch und ein Engagement - auch im Ausland - sollte in jeder Lebensphase möglich sein. Dabei bedarf es auch für Menschen im mittleren und fortgeschrittenen Alter umfassender Qualifizierung durch Vor- und Nachbereitungsangebote sowie unterstützender pädagogischer Begleitung für die Dauer des Dienstes. Eine Kürzung der Einsatzdauer auf einen Zeitraum von 3 bis 6 Monaten oder Angebote im Rahmen von Sabbateinsätzen, die mit den Lebensrealitäten der älteren Menschen übereinstimmen, sollten ebenfalls in die weiteren Überlegungen Eingang finden. 


\section{Corporate Volunteering - betriebliche Freiwilligenprogramme}

Der Vormittag des zweiten Konferenztages stand ganz im Zeichen des so genannten Corporate Volunteerings - einem vergleichsweise jungen Ansatz zur Förderung gesellschaftlichen Engagements in Unternehmen. Prof. Dr. Alexander Thomas (Universität Regensburg), Käthe Engler (IBM, Teilnehmerin an einem Corporate Volunteer Programm) und Pia Himmelsbach (Beraterin, Manager für Menschen $\mathrm{GmbH}$ ) tauschten sich auf dem Podium über die Anreize eines internationalen Engagements für MitarbeiterInnen und Unternehmen aus und eruierten mögliche Potentiale für eine verstärkte Kooperation von zivilen und privaten Akteuren auf diesem Gebiet. Im Rahmen von Angeboten des Corporate Volunteering unterstützen Unternehmen ihre MitarbeiterInnen, innerhalb ihrer Angestelltenverhältnisse ehrenamtlich für gemeinnützige Organisationen bzw. gesellschaftliche Zwecke tätig zu werden. Art und Umfang des Engagements können dabei sehr vielfältig sein und reichen von kurzfristigen Einsätzen, wie z. B. allgemeinen Freiwilligentagen („days of service“), über einwöchige Hospitationen in sozialen Einrichtungen bis hin zu längeren Projekteinsätzen im Ausland (BMFSFJ 2010: 28).

In der Diskussion wurde hervorgehoben, dass die Freistellung von Beschäftigten für einen Einsatz Anreizcharakter besitze und zum Capacity Building bei Organisationen vor Ort beitrage. Ein weiterer Nutzen liege aber auch in der Erschließung neuer Märkte sowie im Bereich der Personalentwicklung. Kommunikations- und Sozialverhalten, Fähigkeiten der kreativen Problemlösung, Team- und Organisationskompetenzen der MitarbeiterInnen werden durch Corporate Volunteering gefördert und können im Anschluss auf die weitere berufliche Arbeit übertragen werden. Gleichzeitig werden Maßnahmen von Unternehmen im Bereich Corporate Volunteering auch als Instrument zur Demonstration von gesellschaftlichem Engagement eingesetzt und in der Regel in eine übergreifende Corporate Social Responsibility ${ }^{3}$ Strategie eingebettet.

Die Diskussion über eine stärkere Zusammenarbeit von Zivilgesellschaft und Privatsektor war zum Teil sehr kontrovers. Zum einen wurden unterschiedliche Beweggründe für gesellschaftliches Engagement - die Rollenreflexion der Freiwilligen auf der einen und funktionales Denken der Corporate Volunteers auf der anderen Seite - gegenüber gestellt, zum anderen die multiperspektivischen Anreize von Unternehmen kritisch hinterfragt.

3 Corporate Social Responsibility (CSR) steht für verantwortliches unternehmerisches Handeln und bezeichnet ein ganzheitliches, alle Dimensionen der Nachhaltigkeit integrierendes Unternehmenskonzept, das die sozialen, ökologischen und ökonomischen Beiträge eines Unternehmens zur Übernahme gesellschaftlicher Verantwortung beinhaltet, die über die Einhaltung gesetzlicher Bestimmungen hinausgehen (Europäische Kommission 2001: 8ff.). 
Pia Himmelsbach führte an, dass auch das Profil der TeilnehmerInnen ein Anderes sei. Beispielsweise nähmen bei vermittelten Einsätzen von „Manager für Menschen “ überwiegend Personen teil, die zuvor noch keine interkulturellen Erfahrungen gesammelt haben und für den entwicklungspolitischen Kontext als weniger sensibilisiert wahrgenommen werden. Berücksichtigt man bisherige Erhebungen zum Profil der Teilnehmenden in internationalen Freiwilligendiensten, sind es aber genau diese Zielgruppen, die durch bestehende Programme noch nicht angesprochen werden, sodass sich positive Synergien für eine Kooperation ergeben können (AKLHÜ 2013: 12, BMZ 2011: 6).

Der Ausbau von Corporate Volunteering, begriffen als unternehmensinternes Bildungsinstrument zur Qualifizierung und Sensibilisierung insbesondere der durch internationale Dienste noch nicht angesprochenen Zielgruppen, könnte hier folglich die entscheidende Brücke für eine Zusammenarbeit sein, in der Unternehmen mit ihren spezifischen Ressourcen Non-Profit-Organisationen unterstützen und diese im Gegenzug Einblicke in ihre jeweiligen Projekt- und Erfahrungswelten anbieten. Aktivitäten des Arbeitgebers in der Betrieblichen Gesundheitsförderung $^{4}$ bieten an dieser Stelle wichtige Anknüpfungspunkte für eine erfolgreiche Zusammenarbeit von zivilgesellschaftlichen und privaten Akteuren, z. B. in der Organisation und Durchführung von Auszeiten oder Social Sabbaticals.

\section{Ausbau und Weiterentwicklung der Incoming-Freiwilligendienste}

„Outgoing gleich Incoming“ - mit dieser Vision leitete Dr. Gisela Kurth (Koordinatorin, Arbeitskreis Lernen und Helfen in Übersee e. V.) zum letzten Teil der Konferenz über, bei dem sich die TeilnehmerInnen in vier Arbeitsgruppen intensiv mit der zukünftigen inhaltlichen und konzeptionellen Ausgestaltung der Aufnahmeprogramme von Freiwilligen in Deutschland auseinandersetzten.

Bereits seit einigen Jahrzehnten vermitteln einzelne Träger aus der Zivilgesellschaft grenzüberschreitend Freiwillige aus dem Ausland. Im Zuge dessen wurden schon zu einem frühen Zeitpunkt von dieser Seite internationale Freiwilligendienste auf Augenhöhe gefordert, die durch eine quantitative Angleichung der Entsende- und Aufnahmezahlen erreicht werden sollten. Das Modellprojekt „Internationale Freiwilligendienste für unterschiedliche Lebensphasen“ (IFL) in den Jahren 2005 bis 2008 ermöglichte schließlich erstmals eine staatliche

4 Ziel der Betrieblichen Gesundheitsförderung ist es, durch gute Arbeitsbedingungen und Lebensqualität am Arbeitsplatz die Belastungen der Beschäftigten zu reduzieren, persönliche Ressourcen zu stärken und Gesundheit und Motivation nachhaltig zu fördern. Der betriebswirtschaftliche Nutzen liegt in der Verringerung von Fehlzeiten und Fluktuationen sowie in der Steigerung von Produktivität, Leistungsbereitschaft und Mitarbeiterzufriedenheit, die wiederum die Attraktivität des Arbeitgebers erhöht (Europäische Kommission 1997: 2ff.). 
Förderung von Incoming-Freiwilligendiensten auf privatrechtlicher Grundlage (zze 2007: 10, 20, 36).

Auch wenn es mit dem Europäischen Freiwilligendienst (EFD) und dem Freiwilligen Sozialen Jahr (FSJ) in Deutschland bereits erste gesetzlich geregelte Engagementmöglichkeiten gab, hat dieses Thema insbesondere in den vergangenen Jahren durch die Teilnahmemöglichkeit von AusländerInnen am 2011 geschaffenen Bundesfreiwilligendienst (BFD) sowie durch die Einführung des (zunächst einseitig konzipierten) weltwärts-Programms im Jahr 2008 politischen Aufwind bekommen: Viele beteiligten Akteure des entwicklungspolitischen Freiwilligendienstes sprachen sich für eine Süd-Nord-Komponente aus, die im Jahr 2013 schließlich eingeführt wurde (Engagement Global 2013: 3f.).

Ein wichtiger Impuls stammt in dieser Diskussion von den Reaktionen der (ehemaligen) Freiwilligen selbst, die sich aufgrund ihrer individuellen Erfahrungen der Privilegierung im globalen Süden für einen gleichberechtigten Austausch stark gemacht haben. Eine quantitative Angleichung scheint jedoch bisher noch weit entfernt: 7.871 deutsche Freiwillige haben im Jahr 2012 ihren Freiwilligendienst im Ausland durchgeführt - demgegenüber stehen 559 (2012) und 625 (2013) Incoming-Freiwilligeneinsätze in Deutschland (AKLHÜ 2014: 8, AKLHÜ 2013: 8). ${ }^{5}$

Nicht nur aus Gründen der Gleichstellung, sondern auch vor dem Hintergrund des demografischen Wandels - im Sinne eines Rückgangs der absoluten Zahlen potentiell (jüngerer) Interessenten für einen Inlandsdienst - stellt die Ansprache von Freiwilligen aus dem Ausland eine Möglichkeit dar, junge Menschen für einen internationalen Dienst in Deutschland zu gewinnen und gesellschaftliches Engagement nachhaltig zu stärken.

Für einen Ausbau der Incoming-Programme ist es zunächst erforderlich, die Erwartungen und Bedürfnisse der Freiwilligen zu eruieren, um daraus Schlussfolgerungen für die weitere inhaltliche und konzeptionelle Ausgestaltung abzuleiten. Viele TeilnehmerInnen sehen in einem Freiwilligendienst die Möglichkeit, sich beruflich zu orientieren und Berufschancen im Heimatland durch den Auslandsaufenthalt zu verbessern, ihre Familie zu unterstützen, Deutschkenntnisse zu erwerben und/oder zu vertiefen, die Lebenswirklichkeit in Deutschland kennenzulernen und ggf. eine Berufs- oder Lebensperspektive in Deutschland aufzubauen.

Wenn Freiwilligendienste als Türöffner für Ausbildungs- und Lebensperspektiven in Deutschland wahrgenommen werden, ist zu überprüfen, ob diese Erwartungen

5 Incoming-Freiwilligendienste können grundsätzlich in zwei Kategorien unterteilt werden: geregelte Freiwilligendienste (G-FD) und Freiwilligendienste auf privatrechtlicher Basis (P-FD). Beide Formen von Freiwilligendiensten sind in dieser Statistik enthalten. Einsätze im Rahmen von Workcamps oder andere Kurzzeiteinsätze bleiben in dieser Berechnung unberücksichtigt, da sie eine eigene Kategorie bilden (AKLHÜ 2014: 5). 
mit den klassischen Zielen der Freiwilligendienste übereinstimmen oder ob bei der weiteren Ausgestaltung der Programme nicht eine stärkere Orientierung an den Lebenswirklichkeiten der Incoming-Freiwilligen vorgenommen werden muss. Des Weiteren sollte bei einer quantitativen Erhöhung und Weiterentwicklung der Incoming-Freiwilligendienste berücksichtigt werden, dass diese nicht als Instrument zur Lösung gesellschaftlicher Aufgaben im Rahmen des Fachkräftemangels eingesetzt werden und die Arbeitsmarktneutralität ${ }^{6}$ gewährleistet ist.

Darüber hinaus wurde in den Arbeitsgruppen der Tagung hervorgehoben, dass Incoming-Freiwillige - die rechtliche Anpassung der Aufenthaltsrechte vorausgesetzt - selbst bestimmen können sollten, ob sie sich nach Ende ihres Dienstes für eine Rückkehr oder ein Weiterleben in Deutschland entscheiden. Die in diesem Zusammenhang häufig artikulierte Befürchtung einer Abwanderung von hochqualifizierten Arbeitskräften, die den Heimatländern Kenntnisse und Fertigkeiten entzieht („Braindrain“), könnte beispielsweise im Rahmen einer langfristig angelegten Studie aufgegriffen werden.

Schließlich sei noch erwähnt, dass die Weiterentwicklung und der Ausbau von Incoming-Freiwilligendiensten insbesondere für die aktuelle Legislaturperiode ein wichtiges politisches Ziel darstellt. ${ }^{7}$ Ein ähnlich positives Bild wurde auch den Ergebnisberichten der MitarbeiterInnen von Trägerorganisationen, Qualitätsstellen und Dachverbänden entnommen, die sich auf der Perspektivkonferenz übergreifend für eine quantitative Angleichung von Entsendung und Aufnahme ausgesprochen haben.

Insgesamt können, sowohl in Bezug auf die rechtlichen und politischen Rahmenbedingungen als auch auf die inhaltliche Ausgestaltung des Incoming-Programms, folgende Empfehlungen als Ergebnisse der Konferenz festgehalten werden:

1. Politische Rahmenbedingungen und finanzielle Ressourcen müssen klar definiert werden, ggfs. auf Basis einer eigenen Förderrichtlinie „Incoming“. Die quantitative Angleichung sollte durch eine sukzessive Steigerung der Aufnahmezahlen pro Jahr erreicht werden.

2. Die inhaltliche und konzeptionelle Weiterentwicklung der Freiwilligenprogramme muss unter Einbeziehung der Lebenswirklichkeiten und

6 Arbeitsmarktneutralität ist immer dann gegeben, wenn ein Dienst die Einstellung von neuen Beschäftigten grundsätzlich nicht verhindert und bestehende Arbeitsplätze nicht substituiert (Klenter 2014: 2ff.).

7 „Wir werden die interkulturelle Öffnung der Freiwilligendienste vorantreiben. (...) Freiwilligendienste sind eine besondere Form des Bürgerschaftlichen Engagements und Bildungsdienste. Wir wollen sie in ihrer bewährten Vielfalt und unter Wahrung ihrer hohen Qualität weiterentwickeln und in zivilgesellschaftlicher Verantwortung ausbauen. (...) Die Freiwilligendienste junger Deutscher im Ausland und von Ausländerinnen und Ausländern, die nach Deutschland kommen, werden wir noch gezielter auf die Interessen und Bedürfnisse der Freiwilligen ausrichten“ (CDU, CSU, SPD 2013: 75ff.). 
individuellen Bedürfnisse der internationalen Freiwilligen erfolgen. Eine Langzeitstudie könnte diese u.a. auf Basis einer Befragung aller bisherigen TeilnehmerInnen ermitteln.

3. Vor dem Hintergrund des Fachkräftemangels und dem Gebot der Arbeitsmarktneutralität müssen in den Einsatzstellen klare Rahmenbedingungen für die Aufnahme von Incoming-Freiwilligen hergestellt und regelmäßig überprüft werden.

4. Die Gefahr der Abwanderung von hochqualifizierten Arbeitskräften, insbesondere spezifischer akademischer Milieus, sollte ebenfalls durch eine wissenschaftliche Studie auf der Basis von Befragungen ehemaliger TeilnehmerInnen statistisch untersucht werden.

5. Ausländische Entsendeorganisationen müssen in den konzeptionellen Weiterentwicklungsprozess eingebunden werden. Entsendung und Aufnahme von Freiwilligen in beide Richtungen muss partnerschaftlich bearbeitet werden. Erwartungen, Bedürfnisse und vorhandene Strukturen der nationalen Entsendeorganisationen sollten in der weiteren konzeptionellen Ausgestaltung Berücksichtigung finden.

\section{Neue Perspektiven für internationale Freiwilligendienste}

An verschiedenen Stellen der Konferenz wurde deutlich, dass angesichts der jüngsten Entwicklungen ein neues Verständnis von internationalen Diensten entsteht, das auch mit einem Bedeutungswandel des Freiwilligenbegriffes einhergeht. Während dieser in seiner ursprünglichen Form bis zum Ende der 1990er Jahre fast ausschließlich für die Bezeichnung eines einjährigen Dienstes von jungen Erwachsenen im In- oder Ausland verwendet wurde, sind in der Vergangenheit neue Modelle internationaler Freiwilligendienste entstanden, die sich im Hinblick auf Zielgruppen, Einsatzfelder, Ausgestaltung und Dauer von den „klassischen“ Diensten unterscheiden. Im Kern zur Erschließung neuer Lebenswelten, der biografischen Orientierung und zum Erwerb sozialer Kompetenzen konzipiert, nehmen Freiwilligendienste heute zunehmend weitere Formen an - als berufliche Auszeit, ehrenamtliches Engagement im Rentenalter oder als Qualifizierungsmöglichkeit im Rahmen der Betrieblichen Gesundheitsförderung. Die Öffnung der Dienste für junge Freiwillige, eine stärkere Teilhabe älterer Menschen, die Förderung von freiwilligem Engagement in Unternehmen sowie der Ausbau der Incoming-Programme sind folglich konkrete Ansätze für eine Weiterentwicklung, die in Teilen schon praktiziert und auch in Zukunft immer größere Bedeutung erfahren wird. Um den Auswirkungen des demografischen Wandels auf die Zielgruppen internationaler Freiwilligendienste zu begegnen und die Folgen der sich verändernden Altersstruktur zu berücksichtigen, gilt es nun, die bisherigen Ergebnisse zu 
konkretisieren und für die weitere Entwicklung klare Bedürfnisse zu formulieren, die Eingang in die politische Agenda finden. Auf dieser Ebene müssen abschließend die rechtlichen sowie finanziellen Rahmenbedingungen geschaffen werden, um eine Teilhabe aller an internationalen Freiwilligendiensten zu ermöglichen.

\section{Literatur}

Arbeitskreis »Lernen und Helfen in Übersee« e.V - AKLHÜ (2013): Freiwillige in internationalen Freiwilligendiensten 2012. Statistische Übersicht 2012, Bonn.

Arbeitskreis »Lernen und Helfen in Übersee« e.V - AKLHÜ (2014): Internationale Freiwillige in Freiwilligendiensten in Deutschland 2012 und 2013. Statistische Übersicht 2012 und 2013, Bonn.

Bundesministerium für Familie, Senioren, Frauen und Jugend - BMFSFJ (2010): Monitor Engagement. Bürgerschaftliches Engagement von Unternehmen in Deutschland. Zwischen Tradition und Innovation, Niestetal.

Bundesministerium für wirtschaftliche Zusammenarbeit und Entwicklung - BMZ (2011): Der entwicklungspolitische Freiwilligendienst „weltwärts”. Kurzfassung der Evaluierung, Berlin.

CDU, CSU, SPD (2013): Deutschlands Zukunft gestalten. Koalitionsvertrag zwischen CDU, CSU und SPD. 18. Legislaturperiode, https://www.cdu.de/sites/default/files/media/dokumente/koalitionsvertrag.pdf (Zugriff am 22. Juni 2014).

Engagement Global gGmbH, Koordinierungsstelle weltwärts (2013): Süd-Nord-Komponente im „Gemeinschaftswerk weltwärts“. Konzept und Informationen zur Kooperation mit dem BFD, http://www.weltwaerts.de/sued-nord.html?file=files/_media/content/Dokumente/4_Uebergreifendes/Ausschreibung_2013_Nord-Sued/Ww_02_Sued-Nord-Konzept_ und_InformationEn_zum_BFD.pdf (Zugriff am 20. Juni 2014).

Europäische Kommission (2001): Europäische Rahmenbedingungen für die soziale Verantwortung der Unternehmen. Grünbuch, Luxemburg.

Europäische Kommission (1997): Luxemburger Deklaration zur Betrieblichen Gesundheitsförderung, Luxemburg.

Klenter, Peter (2014): BBE - 35. Sitzung der AG 3 „Freiwilligendienste“ - Frankfurt a.M. „Arbeitsmarktneutralität“ von Freiwilligendiensten, http://www.b-b-e.de/fileadmin/ inhalte/themen_materialien/pg3_material/Klenter_Arbeitsmarktneutralitaet.pdf (Zugriff am 22. Juni 2014).

Statistisches Bundesamt (2009): Bevölkerung Deutschlands bis 2060. 12. koordinierte Bevölkerungsvorausberechnung. Begleitmaterial zur Pressekonferenz am 18. November 2009 in Berlin, Wiesbaden.

Zentrum für zivilgesellschaftliche Entwicklung - zze (2007): Abschlussbericht der trägerspezifischen Zusatzevaluation der Internationalen Freiwilligendienste für unterschiedliche Lebensphasen (IFL) im Rahmen der „Generationsübergreifenden Freiwilligendienste“ (GüF), http://www.internationale-freiwilligendienste.org/uploads/media/Evaluation_IFL. pdf (Zugriff am 10. August 2014). 